\section{Impact: akin to quantifying dreams}

In our haste to measure everything in order to wring out evidence that non-specialists can understand and to secure funding, we forget that predicting the impact of research is akin to quantifying dreams (Nature 502, 271; 2013). There are no short cuts for proper research assessment.

The impact of research on society is a composite of many strands of work, usually by different scientists and engineers, which - often serendipitously culminate years later in changing some aspect of our lives. But attempting to disentangle those threads is a hopeless task.

There is probably little prognostic value in counting research-paper downloads, for example. In fact, such metrics are but surrogates of real research impact and can generate goals of their own. They encourage 'gaming', or manipulation of data to artificially improve metrics.

When used over time within institutions, metrics can be useful guides - we all need external measures of some sort. It is when they are used as a form of currency in their own right that we get into trouble.

Jim Woodgett LunenfeldTanenbaum Research Institute, Toronto, Canada. woodgett@lunenfeld.ca

\section{Impact: take peer review into account}

Recognition of a researcher's impact should include a measure of his or her contribution as a peer reviewer in maintaining high scientific standards in research papers and grant applications (Nature 502, 287; 2013).

It should be feasible to create a reviewers' equivalent of the $h$-index - which measures the impact of research output in terms of its quantity and popularity. This might indicate, for example, the number and impact of journals and papers for which the reviewer has acted as a referee. Publishers releasing these figures would need to protect the blind or double-blind review process.

They could do this by using a central repository to assign these metrics to particular researchers through identifiers such as the Open Researcher and Contributor ID scheme (see Nature 485, 564; 2012).

As well as securing the reputation of reviewers, such a measure would benefit journals and publishers by encouraging more scientists to undertake refereeing.

E. Tobias Krause Bielefeld University, Germany. tobias.krause@uni-bielefeld.de

\section{Impact: China needs to review its metrics}

Impact metrics are creating their own problems in China (see Nature 502, 271; 2013).

By recognizing only the first and corresponding authors on a paper, for example, China's metrics are indirectly discouraging participation in research consortia. The practice is counterproductive in this era of collaborative science.

Furthermore, review articles do not count towards impact metrics in China, so they do not help to advance an author's academic career. Information overload means that multidisciplinary, scholarly and timely reviews are more in demand than ever. Review authors need comprehensive knowledge, expert insight and outstanding inductive and deductive abilities. We therefore believe that this apparent discrimination is unjustified. Xiangyu Ma College of Preventive Medicine, Third Military Medical University, Chongqing, China.

Zhiyuan Song Southwest Hospital, Third Military Medical University, Chongqing, China. xymacq@hotmail.com

\section{US shutdown should spur other nations}

What the recent US government shutdown has highlighted is just how much it contributes to the world's research infrastructure with PubMed a striking example. By comparison, governments of other high-GDP countries contribute shamefully little.

The world urgently needs freely accessible, searchable, comprehensively referenced and cross-linked international repositories for the burgeoning amounts of untapped 'omics' data (lipidomics being one example). It is time for other wealthy countries to create and fund more such platforms. Julio Licinio, Ma-Li Wong South Australian Health and Medical Research Institute, and Flinders University, Adelaide, Australia.

julio.licinio@sahmri.com

\section{Riding shutdowns in developing world}

The recent US government shutdown hit researchers in the Antarctic particularly hard (G. E. Hofmann Nature 502, 431-432; 2013). But spare a thought for the feats of endurance shown by scientists from the developing world, who continue working in such harsh environments while contending with chronically unpredictable political factors in their own countries.

These scientists regularly battle long-term, governmentinduced setbacks to their research programmes. Regardless of their scientific importance, these studies do not receive widespread publicity when things go wrong - because such events are so frequent. But adverse environmental conditions offer only a limited logistical window for researchers, and natural systems will not wait for the resolution of political brawls.

Examples of such successful long-term research programmes from developing-world scientists include Indian and Brazilian initiatives in the Antarctic (S. D. Gad Curr. Sci. 95, 151; 2008, and P. Artaxo et al. Tellus 44B, 318-334; 1992), and an ongoing subantarctic marinemammal study by South Africans that has so far lasted for 40 years (M. N. Bester et al. Afr. J. Mar. Sci. 33, 511-521; 2011). P. J. Nico de Bruyn University of Pretoria, South Africa. pjndebruyn@zoology.up.ac.za

\section{Science is not about simple stories}

Presenting science as a compelling story is becoming a popular way of communicating results - a technique that is guaranteed to capture the attention of the scientific community and the public. Although science needs great stories, stories are not science.

Storytelling glosses over uncertainties; methodological detail and even results can get lost in the narrative's overarching trajectory. By bounding over hurdles, it keeps the listener rooting for an amazing ending.

But we should not forget that the temptation to package results into a satisfying story has frequently led to the retraction of scientific papers.

Jeroen Bergmann The Brain Sciences Foundation, Rhode Island, and Massachusetts Institute of Technology, USA. jeroen.bergmann@media.mit.edu

\section{CORRECTION}

The Correspondence 'Discard Soviet doctorate systems' (A. Gorobets Nature 503, 39; 2013) wrongly implied that researchers in Russia, Ukraine, Belarus and other former Soviet states do not rely on peerreviewed publications for career advancement. In fact, they do not rely on international peerreviewed publications. Also, doctorate-degree candidates are not classified as 'students'. 